\title{
Bäcklund-Darboux Transformations and Discretizations of Super KdV Equation
}

Ling-Ling XUE and Qing Ping LIU

Department of Mathematics, China University of Mining and Technology, Beijing 100083, P. R. China

E-mail: xue0000@126.com,qpl@cumtb.edu.cn

Received January 02, 2014, in final form April 10, 2014; Published online April 17, 2014

http://dx.doi.org/10.3842/SIGMA.2014.045

\begin{abstract}
For a generalized super KdV equation, three Darboux transformations and the corresponding Bäcklund transformations are constructed. The compatibility of these Darboux transformations leads to three discrete systems and their Lax representations. The reduction of one of the Bäcklund-Darboux transformations and the corresponding discrete system are considered for Kupershmidt's super KdV equation. When all the odd variables vanish, a nonlinear superposition formula is obtained for Levi's Bäcklund transformation for the $\mathrm{KdV}$ equation.
\end{abstract}

Key words: super integrable systems; KdV; Bäcklund-Darboux transformations; discrete integrable systems

2010 Mathematics Subject Classification: 35Q53; 37K10; 35A30

\section{Introduction}

It is well known that the modern theory of integrable systems or soliton theory begins with the study of the celebrated KdV equation by Kruskal and his collaborators [4]. Various types of extensions of this equation exist in literature (see [1] for example) and one of them is the super extensions. The first such extension was proposed by Kupershmidt [7], which reads as

$$
\begin{aligned}
& u_{t}=u_{x x x}-6 u u_{x}+12 \xi_{x x} \xi \\
& \xi_{t}=4 \xi_{x x x}-6 u \xi_{x}-3 u_{x} \xi
\end{aligned}
$$

where subscripts denote partial derivatives, $t$ and $x$ are the temporal variable and spatial variable respectively. $u$ is a bosonic (even or commuting) variable and $\xi$ is a fermionic (odd or anticommuting) variable which fulfill

$$
\xi^{(i)} \xi^{(j)}=-\xi^{(j)} \xi^{(i)}, \quad u^{(i)} u^{(j)}=u^{(j)} u^{(i)}, \quad \xi^{(i)} u^{(j)}=u^{(j)} \xi^{(i)},
$$

where ()$^{(i)}=\partial_{x}{ }^{i}()$. For $\xi=0$, (1) becomes the $\mathrm{KdV}$ equation. Like the KdV equation itself, the super $\mathrm{KdV}$ equation (1), being a bi-Hamiltonian system and possessing Lax representation, is integrable in the conventional sense.

A different super KdV equation was proposed slightly later by Manin and Radul [11] in their study of the supersymmetric KP hierarchy. This system, being the simplest and most important reduction of the supersymmetric KP hierarchy, reads as

$$
\begin{aligned}
& u_{t}=u_{x x x}+6 u u_{x}+3 \xi_{x x} \xi \\
& \xi_{t}=\xi_{x x x}+3(u \xi)_{x} .
\end{aligned}
$$


Even though the above systems (1) and (2) are similar in appearance, they are very different. In fact, as observed by Mathieu [12, 13], the latter is invariant under the following transformation

$$
\widetilde{u}=u+\epsilon \xi_{x}, \quad \widetilde{\xi}=\xi+\epsilon u,
$$

where $\epsilon$ is a fermionic parameter. Then one may introduce a new independent fermionic variable $\theta$ and super field $\alpha=\xi+\theta u$, together with the corresponding super derivative $\mathcal{D}=\partial_{\theta}+\theta \partial_{x}$. In this way, the system (2) may be reformulated as a single equation

$$
\alpha_{t}=\alpha_{x x x}+3(\alpha \mathcal{D} \alpha)_{x} .
$$

For this reason, the system (1) is often referred as the super or fermionic KdV equation, while the system (2) is known as the supersymmetric KdV equation.

Nowadays, discrete integrable systems are very hot topic in the soliton theory, and to construct the discrete versions of the non-commuting extensions of integrable equations is very interesting. Most recently, Grahovski and Mikhailov [5] proposed integrable discretizations for a class of nonlinear Schrödinger equations on Grassmann algebras. Also, with Levi we succeeded in discretizing the supersymmetric KdV equation (2) and both semi-discrete and fully discrete supersymmetric KdV equations are given [19]. The aim of this paper is to study Kupershmidt's super KdV equation (1) in the same spirits. In the following discussion, we will assume that $u$ and $\xi$ depend on not only continuous variables $x$ and $t$, but also are functions of integer-valued variables $n$ and $m$. The subscripts [1] and [2] used in the following denote the shifts of the discrete variables, for example, $\xi_{[1]}=\xi(x, t, n+1, m), \xi_{[2]}=\xi(x, t, n, m+1)$.

The outline of this paper is as follows. In Section 2, we recall a generalized super KdV system and its Lax representation. In Section 3, three different Darboux and Bäcklund transformations are worked out for the generalized super KdV system. Then in Section 4, we employ these transformations to construct discrete integrable super systems and the relevant reductions are discussed. Using two kinds of elementary Darboux transformations, we obtain two differencedifference equations. And by a pair of binary Darboux transformations, we get a differentialdifference equation. The final section summarizes the results.

\section{A generalized super $\mathrm{KdV}$ system}

We aim to construct Darboux and Bäcklund transformations for the super KdV equation (1). To this end, our strategy is to consider a more general super system

$$
\begin{aligned}
& u_{t}=u_{x x x}-6 u u_{x}+6 \xi_{x x} \eta+6 \eta_{x x} \xi \\
& \xi_{t}=4 \xi_{x x x}-6 u \xi_{x}-3 u_{x} \xi \\
& \eta_{t}=4 \eta_{x x x}-6 u \eta_{x}-3 u_{x} \eta
\end{aligned}
$$

where $u=u(x, t)$ is a bosonic variable, $\xi=\xi(x, t)$ and $\eta=\eta(x, t)$ are fermionic variables. To the best of our knowledge, above system was studied first by Holod and Pakuliak [6]. The associated spectral problem is

$$
\begin{array}{ll}
L \psi=\lambda \psi, & L=\partial_{x}^{2}-u-\xi \partial_{x}^{-1} \eta, \\
\psi_{t}=P \psi, & P=4\left(L^{\frac{3}{2}}\right)_{+}=4 \partial_{x}^{3}-6 u \partial_{x}-3 u_{x}-6 \xi \eta .
\end{array}
$$

Introducing $\sigma_{x}=\eta \psi$ and $\chi=\left(\psi, \psi_{x}, \sigma\right)^{\mathrm{T}}$, then we may rewrite (4) in matrix form, that is,

$$
\chi_{x}=\mathcal{L} \chi, \quad \mathcal{L}=\left(\begin{array}{ccc}
0 & 1 & 0 \\
\lambda+u & 0 & \xi \\
\eta & 0 & 0
\end{array}\right)
$$




$$
\chi_{t}=\mathcal{P} \chi, \quad \mathcal{P}=\left(\begin{array}{ccc}
u_{x}-2 \xi \eta & 4 \lambda-2 u & 4 \xi_{x} \\
Z & -u_{x}-2 \xi \eta & 4 \xi_{x x}+(4 \lambda-2 u) \xi \\
4 \eta_{x x}+(4 \lambda-2 u) \eta & -4 \eta_{x} & -4 \xi \eta
\end{array}\right),
$$

where

$$
Z \equiv u_{x x}+(\lambda+u)(4 \lambda-2 u)+2\left(\xi_{x} \eta-\xi \eta_{x}\right)
$$

A direct calculation shows that the Lax equation

$$
L_{t}=[P, L],
$$

or the zero curvature condition

$$
\mathcal{L}_{t}-\mathcal{P}_{x}=[\mathcal{P}, \mathcal{L}]
$$

gives the generalized super KdV system (3).

Remark. For $\xi=\eta$, (3) reduces to Kupershmidt's super KdV equation (1).

Remark. For all fermionic variables disappear, (3) reduces to the KdV equation with linear spectral problem

$$
\chi_{x}=\mathcal{L} \chi, \quad \mathcal{L}=\left(\begin{array}{cr}
0 & 1 \\
\lambda+u & 0
\end{array}\right), \quad \chi_{t}=\mathcal{P} \chi, \quad \mathcal{P}=\left(\begin{array}{cc}
u_{x} & 4 \lambda-2 u \\
u_{x x}+(\lambda+u)(4 \lambda-2 u) & -u_{x}
\end{array}\right) .
$$

In the following, we will use (5) to construct Bäcklund and Darboux transformations.

\section{Darboux and Bäcklund transformations}

Now we manage to construct Darboux and Bäcklund transformations for the generalized super $\mathrm{KdV}$ system (3). For convenience, we introduce the potentials $w$ and $w_{[i]}$ such that $u=w_{x}$, $u_{[i]}=w_{[i]_{x}}$, and define $v_{i}=w_{[i]}-w$ for $i=1,2$. Also, suppose that $\chi_{[0]}=\left(\psi_{[0]}, \psi_{[0]_{x}}, \sigma_{[0]}\right)^{\mathrm{T}}$ is a solution of (5) for $\lambda=p_{1}$, then we find three Darboux transformations and their corresponding Bäcklund transformations, which are listed below.

Case 1. Define

$$
v_{1} \equiv-2\left(\ln \psi_{[0]}\right)_{x}, \quad \xi_{[1]} \equiv \xi_{x}+\frac{1}{2} v_{1} \xi, \quad \eta_{[1]} \equiv-\frac{\sigma_{[0]}}{\psi_{[0]}}
$$

and

$$
\chi_{[1]} \equiv \mathcal{W} \chi, \quad \mathcal{W}=\left(\begin{array}{ccc}
\frac{1}{2} v_{1} & 1 & 0 \\
\lambda-p_{1}+\frac{1}{4} v_{1}^{2}+\xi \eta_{[1]} & \frac{1}{2} v_{1} & \xi \\
\eta_{[1]} & 0 & 1
\end{array}\right)
$$

then $\chi_{[1]}$ satisfies

$$
\chi_{[1]_{x}}=\mathcal{L}_{[1]} \chi_{[1]}, \quad \mathcal{L}_{[1]}=\left(\begin{array}{ccc}
0 & 1 & 0 \\
\lambda+u_{[1]} & 0 & \xi_{[1]} \\
\eta_{[1]} & 0 & 0
\end{array}\right) .
$$

The compatibility of the two linear systems (6) and (7) yields

$$
\mathcal{W}_{x}+\mathcal{W} \mathcal{L}-\mathcal{L}_{[1]} \mathcal{W}=0
$$

which leads to a Bäcklund transformation

$$
\xi_{[1]}=\xi_{x}+\frac{1}{2} v_{1} \xi, \quad \eta=-\eta_{[1]_{x}}+\frac{1}{2} v_{1} \eta_{[1]}, \quad w_{[1]_{x}}=-w_{x}+\frac{1}{2} v_{1}^{2}-2 p_{1}+2 \xi \eta_{[1]} .
$$


Remark. During the 5th International Workshop on Nonlinear Mathematical Physics and the 12th National Conference on Integrable Systems, held in Hangzhou last summer, we learnt that professor R.G. Zhou also considered such Darboux transformation [20].

Case 2. Define

$$
\bar{\sigma}_{[0]_{x}}=\xi \psi_{[0]}, \quad v_{1} \equiv-2\left(\ln \psi_{[0]}\right)_{x}+2 \frac{\bar{\sigma}_{[0]} \sigma_{[0]}}{\psi_{[0]}^{2}}, \quad \xi_{[1]} \equiv \frac{\bar{\sigma}_{[0]}}{\psi_{[0]}}, \quad \eta_{[1]} \equiv-\eta_{x}-\frac{1}{2} v_{1} \eta
$$

and

$$
\chi_{[1]} \equiv \mathcal{W} \chi, \quad \mathcal{W}=\left(\begin{array}{ccc}
\frac{1}{2} v_{1} & 1 & -\xi_{[1]} \\
\lambda-p_{1}+\frac{1}{4} v_{1}^{2} & \frac{1}{2} v_{1} & -\frac{1}{2} v_{1} \xi_{[1]} \\
-\frac{1}{2} v_{1} \eta & -\eta & \lambda-p_{1}-\xi_{[1]} \eta
\end{array}\right)
$$

then $\chi_{[1]}$ satisfies

$$
\chi_{[1]_{x}}=\mathcal{L}_{[1]} \chi_{[1]} .
$$

The compatibility of the linear systems (9) and (10) supplies

$$
\mathcal{W}_{x}+\mathcal{W} \mathcal{L}-\mathcal{L}_{[1]} \mathcal{W}=0
$$

which gives the following Bäcklund transformation

$$
\xi=\xi_{[1]_{x}}-\frac{1}{2} v_{1} \xi_{[1]}, \quad \eta_{[1]}=-\eta_{x}-\frac{1}{2} v_{1} \eta, \quad w_{[1]_{x}}=-w_{x}+\frac{1}{2} v_{1}^{2}-2 p_{1}+2 \xi_{[1]} \eta .
$$

Remark. For all fermionic variables vanish, it is easy to see that above Darboux transformations and Bäcklund transformations reduce to the well-known results for the KdV equation.

Remark. We observe that there exists a symmetry between Bäcklund transformation (8) and Bäcklund transformation (11). In fact, if we make the following replacements:

$$
\xi \leftrightarrow \xi_{[1]}, \quad \eta \leftrightarrow \eta_{[1]}, \quad w \leftrightarrow w_{[1]},
$$

then (8) becomes (11).

Case 3. Define

$$
\begin{array}{ll}
\bar{\sigma}_{[0]_{x}}=\xi \psi_{[0]}, & F_{x}=\psi_{[0]}^{2}+\frac{F}{\psi_{[0]}^{2}} \bar{\sigma}_{[0]} \sigma_{[0]}, \\
v_{1} \equiv-2 \frac{\psi_{[0]}^{2}}{F}, & \xi_{[1]} \equiv \xi-\frac{\psi_{[0]}}{F} \bar{\sigma}_{[0]}, \quad \eta_{[1]} \equiv \eta-\frac{\psi_{[0]}}{F} \sigma_{[0]}
\end{array}
$$

and

$$
\begin{aligned}
\chi_{[1]} & \equiv \mathcal{W} \chi, \\
\mathcal{W} & =\left(\begin{array}{ccc}
\lambda+A & \frac{1}{2} v_{1} & \xi-\xi_{[1]} \\
\frac{1}{2} \lambda v_{1}+B & \lambda+A+\frac{v_{1 x}}{2} & -\left(\frac{v_{1}}{4}+\frac{v_{1 x}}{2 v_{1}}\right)\left(\xi_{[1]}-\xi\right) \\
\left(\frac{v_{1}}{4}-\frac{v_{1 x}}{2 v_{1}}\right)\left(\eta_{[1]}-\eta\right) & \eta_{[1]}-\eta & \lambda-p_{1}+\frac{2}{v_{1}}\left(\xi_{[1]}-\xi\right)\left(\eta_{[1]}-\eta\right)
\end{array}\right)
\end{aligned}
$$

with

$$
\begin{aligned}
& A \equiv-p_{1}-\frac{v_{1 x}}{4}+\frac{v_{1}^{2}}{8}+\frac{1}{v_{1}}\left(\xi_{[1]}-\xi\right)\left(\eta_{[1]}-\eta\right), \\
& B \equiv-\frac{v_{1 x}^{2}}{8 v_{1}}+\frac{v_{1}^{3}}{32}-\frac{p_{1}}{2} v_{1}+\frac{1}{2}\left(\xi_{[1]}-\xi\right)\left(\eta_{[1]}-\eta\right),
\end{aligned}
$$


then $\chi_{[1]}$ solves

$$
\chi_{[1]_{x}}=\mathcal{L}_{[1]} \chi_{[1]} .
$$

Similarly, the compatibility of the above linear systems leads to

$$
\mathcal{W}_{x}+\mathcal{W} \mathcal{L}-\mathcal{L}_{[1]} \mathcal{W}=0,
$$

or the Bäcklund transformation

$$
\begin{aligned}
& \xi_{[1]_{x}}=\xi_{x}+\frac{v_{1}}{2} \xi+\left(\frac{v_{1}}{4}+\frac{v_{1 x}}{2 v_{1}}\right)\left(\xi_{[1]}-\xi\right), \\
& \eta_{[1]_{x}}=\eta_{x}+\frac{v_{1}}{2} \eta+\left(\frac{v_{1}}{4}+\frac{v_{1 x}}{2 v_{1}}\right)\left(\eta_{[1]}-\eta\right), \\
& w_{[1]_{x x}}=w_{x x}+v_{1}\left(v_{1 x}+2 w_{x}\right)+\frac{v_{1 x}{ }^{2}}{2 v_{1}}-\frac{v_{1}^{3}}{8}+2 p_{1} v_{1}+2\left(\eta \xi_{[1]}+\xi \eta_{[1]}\right) .
\end{aligned}
$$

It is remarked that in the last case if the fermionic variables $\xi$ and $\eta$ vanish, we recover a Darboux transformation and related Bäcklund transformation for the KdV equation, which are nothing but the ones obtained by Levi [9]. Such a Darboux transformation is also known as binary Darboux transformation in literature [14]. Explicitly, the related Bäcklund transformation [9] reads as

$$
w_{[1]_{x x}}=w_{x x}+v_{1}\left(v_{1 x}+2 w_{x}\right)+\frac{v_{1 x}^{2}}{2 v_{1}}-\frac{v_{1}^{3}}{8}+2 p_{1} v_{1} .
$$

Darboux transformations of binary type may be regarded as the composition of elementary Darboux transformations [16] (see also [3]). Thus it is natural to expect that Levi's Bäcklund transformation (13) is also the composition of elementary Bäcklund transformations and this is indeed the case. To see this we consider two copies of elementary Bäcklund transformation for the KdV equation, namely

$$
\left(w_{[1]}+\bar{w}\right)_{x}=\frac{1}{2}\left(w_{[1]}-\bar{w}\right)^{2}-2 p_{1}, \quad(w+\bar{w})_{x}=\frac{1}{2}(\bar{w}-w)^{2}-2 p_{2},
$$

then, eliminating $\bar{w}$ leads to

$$
w_{[1]_{x x}}=w_{x x}+v_{1}\left(v_{1 x}+2 w_{x}\right)+\frac{v_{1 x}^{2}}{2 v_{1}}-\frac{v_{1}^{3}}{8}+\left(p_{1}+p_{2}\right) v_{1}-\frac{2}{v_{1}}\left(p_{1}-p_{2}\right)^{2},
$$

which reduces to (13) if $p_{1}=p_{2}$. Similar idea works for the super case and we can obtain the Bäcklund transformation (12) by the superposition of the elementary Bäcklund transformations (8) or (11). Thus, elementary Darboux/Bäcklund transformations are more fundamental and binary Darboux/Bäcklund transformations are more involved. The point is that while it is not clear how to reduce the Darboux/Bäcklund transformations of the Cases 1 and 2 to Kupershmidt's super KdV equation (1), the reduction is feasible and easy to implement for the last case. Indeed, for $\xi=\eta$, define

$$
F_{x}=\psi_{[0]}^{2}, \quad v_{1} \equiv-2(\ln F)_{x}, \quad \xi_{[1]} \equiv \xi-\frac{\psi_{[0]}}{F} \sigma_{[0]}
$$

and

$$
\chi_{[1]} \equiv \mathcal{W} \chi, \quad \mathcal{W}=\left(\begin{array}{ccc}
\lambda+A & \frac{1}{2} v_{1} & \xi-\xi_{[1]} \\
\frac{1}{2} \lambda v_{1}+B & \lambda+A+\frac{v_{1 x}}{2} & -\left(\frac{v_{1}}{4}+\frac{v_{1 x}}{2 v_{1}}\right)^{\left(\xi_{[1]}-\xi\right)} \\
\left(\frac{v_{1}}{4}-\frac{v_{1 x}}{2 v_{1}}\right)\left(\xi_{[1]}-\xi\right) & \xi_{[1]}-\xi & \lambda-p_{1}
\end{array}\right)
$$


with

$$
A \equiv-p_{1}-\frac{v_{1 x}}{4}+\frac{v_{1}^{2}}{8}, \quad B \equiv-\frac{v_{1 x^{2}}}{8 v_{1}}+\frac{v_{1}^{3}}{32}-\frac{p_{1}}{2} v_{1},
$$

then it is straightforward to check that $\chi_{[1]}$ satisfies

$$
\chi_{[1]_{x}}=\mathcal{L}_{[1]} \chi_{[1]} .
$$

The corresponding Bäcklund transformation is

$$
\begin{aligned}
& \xi_{[1]_{x}}=\xi_{x}+\frac{v_{1}}{2} \xi+\left(\frac{v_{1}}{4}+\frac{v_{1 x}}{2 v_{1}}\right)\left(\xi_{[1]}-\xi\right), \\
& w_{[1]_{x x}}=w_{x x}+v_{1}\left(v_{1 x}+2 w_{x}\right)+\frac{v_{1 x}^{2}}{2 v_{1}}-\frac{v_{1}^{3}}{8}+2 p_{1} v_{1}+4 \xi \xi_{[1]} .
\end{aligned}
$$

Thus, we obtain a Bäcklund transformation for Kupershmidt's super KdV equation (1).

\section{Discrete systems}

Integrable discretizations have been studied extensively since the seventies of last century and various approaches have been proposed (see [15, 18]). Among them, the method, based on Darboux and Bäcklund transformations, which first appeared in [8, 10], has been proved to be very fruitful. This idea is also applicable to super integrable systems and supersymmetric integrable systems [5, 19]. We now adopt this idea for the super KdV equation (3) and its reduction - Kupershmidt's super KdV equation to construct their discrete counterparts.

We start our consideration with Darboux transformations presented in the first two cases of last section.

Case A. Consider a pair of Darboux transformations which are given in Case 1:

$$
\begin{aligned}
& \chi_{[1]}=\mathcal{W} \chi, \quad \mathcal{W}=\left(\begin{array}{ccc}
\frac{1}{2} v_{1} & 1 & 0 \\
\lambda-p_{1}+\frac{1}{4} v_{1}^{2}+\xi \eta_{[1]} & \frac{1}{2} v_{1} & \xi \\
\eta_{[1]} & 0 & 1
\end{array}\right), \\
& \frac{1}{2} v_{2} \\
& \chi_{[2]}=\mathcal{N} \chi, \quad \mathcal{N}=\left(\begin{array}{ccc}
1 & 0 \\
\lambda-p_{2}+\frac{1}{4} v_{2}^{2}+\xi \eta_{[2]} & \frac{1}{2} v_{2} & \xi \\
\eta_{[2]} & 0 & 1
\end{array}\right) .
\end{aligned}
$$

Now the compatibility condition of (15) and (16), namely

$$
\mathcal{W}_{[2]} \mathcal{N}=\mathcal{N}_{[1]} \mathcal{W}
$$

yields an integrable difference-difference system

$$
\begin{aligned}
& \xi_{[2]}=\xi_{[1]}+\frac{2\left(p_{2}-p_{1}\right)}{w_{[12]}-w} \xi, \quad \eta_{[2]}=\eta_{[1]}+\frac{2\left(p_{1}-p_{2}\right)}{w_{[12]}-w} \eta_{[12]}, \\
& w_{[2]}=w_{[1]}+\frac{4\left(p_{2}-p_{1}\right)}{w_{[12]}-w}+\frac{8\left(p_{2}-p_{1}\right)}{\left(w_{[12]}-w\right)^{2}} \xi \eta_{[12]} .
\end{aligned}
$$

Case B. Consider two Darboux transformations which are obtained in Cases 1 and 2 respectively:

$$
\chi_{[1]}=\mathcal{W} \chi, \quad \mathcal{W}=\left(\begin{array}{ccc}
\frac{1}{2} v_{1} & 1 & 0 \\
\lambda-p_{1}+\frac{1}{4} v_{1}^{2}+\xi \eta_{[1]} & \frac{1}{2} v_{1} & \xi \\
\eta_{[1]} & 0 & 1
\end{array}\right),
$$




$$
\chi_{[2]}=\mathcal{M} \chi, \quad \mathcal{M}=\left(\begin{array}{ccc}
\frac{1}{2} v_{2} & 1 & -\xi_{[2]} \\
\lambda-p_{2}+\frac{1}{4} v_{2}^{2} & \frac{1}{2} v_{2} & -\frac{1}{2} v_{2} \xi_{[2]} \\
-\frac{1}{2} v_{2} \eta & -\eta & \lambda-p_{2}-\xi_{[2]} \eta
\end{array}\right)
$$

Now the compatibility condition of (18) and (19),

$$
\mathcal{W}_{[2]} \mathcal{M}=\mathcal{M}_{[1]} \mathcal{W}
$$

provides us the following integrable difference-difference system

$$
\begin{gathered}
\xi_{[12]}=\xi+\frac{2\left(p_{1}-p_{2}\right)}{w_{[1]}-w_{[2]}} \xi_{[2]}, \quad \eta_{[21]}=\eta+\frac{2\left(p_{2}-p_{1}\right)}{w_{[1]}-w_{[2]}} \eta_{[1]}, \\
w_{[12]}=w_{[21]}=w+\frac{4\left(p_{1}-p_{2}\right)}{w_{[1]}-w_{[2]}}+\frac{8\left(p_{1}-p_{2}\right)}{\left(w_{[1]}-w_{[2]}\right)^{2}} \xi_{[2]} \eta_{[1]} .
\end{gathered}
$$

While for the bosonic field we have $w_{[12]}=w_{[21]}$, it is not clear from the equations (20) that whether the same situation appears for the fermionic variables $\xi$ and $\eta$. We now show that this is indeed the case. By means of the Bäcklund transformations

$$
\begin{aligned}
\eta_{[1]_{x}}=-\eta+\frac{1}{2}\left(w_{[1]}-w\right) \eta_{[1]}, & \xi_{[21]}=\xi_{[2]_{x}}+\frac{1}{2}\left(w_{[21]}-w_{[2]}\right) \xi_{[2]}, \\
\xi_{[2]_{x}}=\xi+\frac{1}{2}\left(w_{[2]}-w\right) \xi_{[2]}, & \eta_{[12]}=-\eta_{[1]_{x}}-\frac{1}{2}\left(w_{[12]}-w_{[1]}\right) \eta_{[1]},
\end{aligned}
$$

it follows that

$$
\xi_{[21]}=\xi+\frac{1}{2}\left(w_{[21]}-w\right) \xi_{[2]}, \quad \eta_{[12]}=\eta-\frac{1}{2}\left(w_{[12]}-w\right) \eta_{[1]},
$$

these equations, taking (20) into account, yield

$$
\xi_{[21]}=\xi+\frac{2\left(p_{1}-p_{2}\right)}{w_{[1]}-w_{[2]}} \xi_{[2]}, \quad \eta_{[12]}=\eta-\frac{2\left(p_{1}-p_{2}\right)}{w_{[1]}-w_{[2]}} \eta_{[1]}
$$

thus

$$
\xi_{[21]}=\xi_{[12]}, \quad \eta_{[12]}=\eta_{[21]} .
$$

Remark. For all fermionic fields vanish, both (17) and (20) reduce to

$$
w_{[12]}=w+\frac{4\left(p_{1}-p_{2}\right)}{w_{[1]}-w_{[2]}},
$$

which is the potential KdV lattice or H1 in Adler-Bobenko-Suris's classification [2] or the classical nonlinear superposition formula for the $\mathrm{KdV}$ equation.

Finally we consider

Case C. Assume a pair of Darboux transformations which are provided in Case 3:

$$
\begin{aligned}
& \chi_{[1]}=\mathcal{W} \chi, \\
& \chi_{[2]}=\mathcal{V} \chi,
\end{aligned}
$$

where

$$
\mathcal{W}=\left(\begin{array}{ccc}
\lambda+A & \frac{1}{2} v_{1} & \xi-\xi_{[1]} \\
\frac{1}{2} \lambda v_{1}+B & \lambda+A+\frac{v_{1 x}}{2} & -\left(\frac{v_{1}}{4}+\frac{v_{1 x}}{2 v_{1}}\right)\left(\xi_{[1]}-\xi\right) \\
\left(\frac{v_{1}}{4}-\frac{v_{1 x}}{2 v_{1}}\right)\left(\eta_{[1]}-\eta\right) & \eta_{[1]}-\eta & \lambda-p_{1}+\frac{2}{v_{1}}\left(\xi_{[1]}-\xi\right)\left(\eta_{[1]}-\eta\right)
\end{array}\right)
$$


with

$$
\begin{aligned}
A & \equiv-p_{1}-\frac{v_{1 x}}{4}+\frac{v_{1}^{2}}{8}+\frac{1}{v_{1}}\left(\xi_{[1]}-\xi\right)\left(\eta_{[1]}-\eta\right), \\
B & \equiv-\frac{v_{1 x}^{2}}{8 v_{1}}+\frac{v_{1}^{3}}{32}-\frac{p_{1}}{2} v_{1}+\frac{1}{2}\left(\xi_{[1]}-\xi\right)\left(\eta_{[1]}-\eta\right),
\end{aligned}
$$

the matrix $\mathcal{V}$ is the matrix $\mathcal{W}$ with $p_{1}, \xi_{[1]}, \eta_{[1]}$ and $w_{[1]}$ replaced by $p_{2}, \xi_{[2]}, \eta_{[2]}$ and $w_{[2]}$ respectively. By the compatibility $\left(\chi_{[1]}\right)_{[2]}=\left(\chi_{[2]}\right)_{[1]}$ of $(21)$ and (22), i.e. the Bianchi permutability of the Bäcklund transformation (8) with $\xi_{[21]}=\xi_{[12]}, \eta_{[21]}=\eta_{[12]}$ and $w_{[21]}=w_{[12]}$, we find that the following consistency condition

$$
\mathcal{W}_{[2]} \mathcal{V}=\mathcal{V}_{[1]} \mathcal{W}
$$

must be true. (23) leads to

$$
\begin{aligned}
w_{[12]_{x}}= & w_{[2]_{x}}+\frac{1}{2}\left(w_{[12]}-w_{[2]}\right)^{2}+\left(w_{[12]}-w_{[2]}\right)\left[\frac{1}{2}\left(w_{[2]}-w_{[1]}\right)+\left(\ln \left(w_{[1]}-w_{[2]}\right)\right)_{x}\right] \\
& -\left(w_{[1]}-w_{[2]}\right)^{-1}\left[\frac{1}{2} h+4 c\left(w_{[12]}-w_{[2]}-w_{[1]}+w\right)\right] \\
& -4\left[v_{1} v_{2}\left(w_{[12]}-w_{[2]}\right)\left(w_{[1]}-w_{[2]}\right)\left(w_{[12]}-w_{[1]}\right)\right]^{-1} \\
& \times\left\{-v_{2}\left(w_{[12]}-w_{[2]}\right)\left(w_{[12]}-w\right)^{2}\left(\xi_{[1]}-\xi\right)\left(\eta_{[1]}-\eta\right)\right. \\
& -v_{1} v_{2}\left(w_{[12]}-w\right)\left(w_{[1]}-w_{[2]}\right)\left(\xi_{[12]}-\xi_{[2]}\right)\left(\eta_{[12]}-\eta_{[2]}\right) \\
& +v_{1}\left(w_{[12]}-w_{[2]}\right)\left(w_{[12]}-w\right)\left(w_{[12]}-w_{[2]}-v_{1}\right)\left(\xi_{[2]}-\xi\right)\left(\eta_{[2]}-\eta\right) \\
& +v_{1}^{2} v_{2}\left(w_{[12]}-w_{[2]}\right)\left[\left(\xi_{[1]}-\xi\right)\left(\eta_{[12]}-\eta\right)-\left(\xi_{[2]}-\xi\right)\left(\eta_{[12]}-\eta_{[2]}\right)\right] \\
& +v_{1} v_{2}\left(w_{[12]}-w_{[2]}\right)\left(w_{[1]}+w-2 w_{[12]}\right) \\
& \left.\times\left[\left(\eta_{[1]}-\eta\right)\left(\xi_{[12]}-\xi\right)-\left(\eta_{[2]}-\eta\right)\left(\xi_{[12]}-\xi_{[2]}\right)\right]\right\}
\end{aligned}
$$

and

$$
\begin{aligned}
\xi_{[12]}= & \xi+f_{1}\left(\xi_{1}-\xi\right)+f_{2}\left(\xi_{2}-\xi\right)+\left(\xi_{1}-\xi\right)\left(\xi_{2}-\xi\right)\left[f_{3}\left(\eta_{1}-\eta\right)+f_{4}\left(\eta_{2}-\eta\right)\right], \\
\eta_{[12]}= & \eta+f_{1}\left(\eta_{1}-\eta\right)+f_{2}\left(\eta_{2}-\eta\right)+\left(\eta_{1}-\eta\right)\left(\eta_{2}-\eta\right)\left[f_{3}\left(\xi_{1}-\xi\right)+f_{4}\left(\xi_{2}-\xi\right)\right], \\
w_{[12]}= & w+g_{1}+g_{2}\left[\left(\xi_{1}-\xi\right)\left(\eta_{2}-\eta\right)+\left(\eta_{1}-\eta\right)\left(\xi_{2}-\xi\right)\right] \\
& +g_{3}\left(\xi_{1}-\xi\right)\left(\xi_{2}-\xi\right)\left(\eta_{1}-\eta\right)\left(\eta_{2}-\eta\right),
\end{aligned}
$$

where

$$
\begin{aligned}
& c \equiv p_{1}-p_{2}, \quad h \equiv v_{1} v_{2}\left(v_{1}-v_{2}\right)+2\left(v_{1} v_{2 x}-v_{2} v_{1 x}\right), \\
& f_{1} \equiv \frac{8 c v_{2}\left(h-8 c v_{1}\right)}{h^{2}-64 c^{2} v_{1} v_{2}}, \quad f_{2} \equiv \frac{8 c v_{1}\left(h-8 c v_{2}\right)}{h^{2}-64 c^{2} v_{1} v_{2}}, \\
& f_{3} \equiv \frac{-64 c v_{2}}{\left(h^{2}-64 c^{2} v_{1} v_{2}\right)^{2}}\left[h^{2}-16 c h v_{1}+64 c^{2} v_{1} v_{2}\right], \\
& f_{4} \equiv \frac{-64 c v_{1}}{\left(h^{2}-64 c^{2} v_{1} v_{2}\right)^{2}}\left[h^{2}-16 c h v_{2}+64 c^{2} v_{1} v_{2}\right], \\
& g_{1} \equiv \frac{16 c v_{1} v_{2}}{h^{2}-64 c^{2} v_{1} v_{2}}\left[h-4 c\left(v_{1}+v_{2}\right)\right], \quad g_{2} \equiv \frac{128 c v_{1} v_{2}}{\left(h^{2}-64 c^{2} v_{1} v_{2}\right)^{2}}\left[h-8 c v_{1}\right]\left[h-8 c v_{2}\right], \\
& g_{3} \equiv \frac{2048 c v_{1} v_{2}}{\left(h^{2}-64 c^{2} v_{1} v_{2}\right)^{3}}\left[-h^{3}+12 c h^{2}\left(v_{1}+v_{2}\right)-192 c^{2} h v_{1} v_{2}+256 c^{3} v_{1} v_{2}\left(v_{1}+v_{2}\right)\right] .
\end{aligned}
$$


Here the system (24) serves as a discrete system which, being cumbersome, nevertheless has the advantage that it is easy to handle if one considers the reductions. In fact, we have two interesting cases:

1. For $\xi=\eta$, the consistency condition (23) leads to

$$
\begin{aligned}
w_{[12]_{x}}= & w_{[2]_{x}}+\frac{1}{2}\left(w_{[12]}-w_{[2]}\right)^{2}+\left(w_{[12]}-w_{[2]}\right)\left[\frac{1}{2}\left(w_{[2]}-w_{[1]}\right)+\left(\ln \left(w_{[1]}-w_{[2]}\right)\right)_{x}\right] \\
& -\frac{h+8 c\left(w_{[12]}-w_{[2]}-w_{[1]}+w\right)+16\left(\xi_{[12]}-\xi\right)\left(\xi_{[1]}-\xi_{[2]}\right)}{2\left(w_{[1]}-w_{[2]}\right)},
\end{aligned}
$$

and

$$
\xi_{[12]}=\xi+f_{1}\left(\xi_{1}-\xi\right)+f_{2}\left(\xi_{2}-\xi\right), \quad w_{[12]}=w+g_{1}+2 g_{2}\left(\xi_{1}-\xi\right)\left(\xi_{2}-\xi\right) .
$$

By a direct calculation, one can check that (25) is satisfied if we substitute (26) into it and take account of the corresponding Bäcklund transformations (14) into consideration.

2. For all fermionic fields vanish, (23) leads to

$$
\begin{aligned}
w_{[12]_{x}}= & w_{[2]_{x}}+\left(w_{[12]}-w_{[2]}\right)\left[\frac{1}{2}\left(w_{[2]}-w_{[1]}\right)+\left(\ln \left(w_{[1]}-w_{[2]}\right)\right)_{x}\right] \\
& +\frac{1}{2}\left(w_{[12]}-w_{[2]}\right)^{2}-\frac{h+8 c\left(w_{[12]}-w_{[2]}-w_{[1]}+w\right)}{2\left(w_{[1]}-w_{[2]}\right)}
\end{aligned}
$$

and

$$
w_{[12]}=w+g_{1}
$$

Also, a direct calculation shows that (27) is satisfied if we substitute (28) into it and take the corresponding Bäcklund transformations (13) into consideration. Thus (28) may be regarded as the nonlinear superposition formula for the Bäcklund transformation (13) for the KdV equation. To the best of our knowledge, this result is also new.

\section{Conclusion}

In this paper, we have constructed three types of Bäcklund and Darboux transformations for a generalized super KdV equation. By means of these transformations, the super KdV equation has been discretized. In particular, by considering the reductions, we have succeeded in obtaining a Bäcklund transformation and a discrete version for Kupershmidt's super KdV equation. As a by-product, we have found a nonlinear superposition formula for the Bäcklund transformation obtained by Levi early. The discretization for the supersymmetric Schrödinger equation [17] is under investigated and will appear elsewhere.

\section{Acknowledgments}

The comments of the anonymous referees have been useful in clarifying certain points such as the connection between Levi's Bäcklund transformation and elementary Bäcklund transformation of the KdV equation. This work is supported by the National Natural Science Foundation of China (grant numbers: 10971222, 11271366 and 11331008) and the Fundamental Research Funds for Central Universities. 


\section{References}

[1] Ablowitz M.J., Clarkson P.A., Solitons, nonlinear evolution equations and inverse scattering, London Mathematical Society Lecture Note Series, Vol. 149, Cambridge University Press, Cambridge, 1991.

[2] Adler V.E., Bobenko A.I., Suris Yu.B., Classification of integrable equations on quad-graphs. The consistency approach, Comm. Math. Phys. 233 (2003), 513-543, nlin.SI/0202024.

[3] Cieśliński J.L., Algebraic construction of the Darboux matrix revisited, J. Phys. A: Math. Theor. 42 (2009), 404003, 40 pages, arXiv:0904.3987.

[4] Gardner C.S., Greene J.M., Kruskal M.D., Miura R.M., Method for solving the Korteweg-de Vries equation, Phys. Rev. Lett. 19 (1967), 1095-1097.

[5] Grahovski G.G., Mikhailov A.V., Integrable discretisations for a class of nonlinear Schrödinger equations on Grassmann algebras, Phys. Lett. A 377 (2013), 3254-3259, arXiv:1303.1853.

[6] Holod P.I., Pakuliak S.Z., On the superextension of the Kadomtsev-Petviashvili equation and finite-gap solutions of Korteweg-de Vries superequations, in Problems of Modern Quantum Field Theory (Alushta, 1989), Res. Rep. Phys., Springer, Berlin, 1989, 107-116.

[7] Kupershmidt B.A., A super Korteweg-de Vries equation: an integrable system, Phys. Lett. A 102 (1984), 213-215.

[8] Levi D., Nonlinear differential-difference equations as Bäcklund transformations, J. Phys. A: Math. Gen. 14 (1981), 1083-1098.

[9] Levi D., On a new Darboux transformation for the construction of exact solutions of the Schrödinger equation, Inverse Problems 4 (1988), 165-172.

[10] Levi D., Benguria R., Bäcklund transformations and nonlinear differential difference equations, Proc. Nat. Acad. Sci. USA 77 (1980), 5025-5027.

[11] Manin Yu.I., Radul A.O., A supersymmetric extension of the Kadomtsev-Petviashvili hierarchy, Comm. Math. Phys. 98 (1985), 65-77.

[12] Mathieu P., Superconformal algebra and supersymmetric Korteweg-de Vries equation, Phys. Lett. B 203 (1988), 287-291.

[13] Mathieu P., Supersymmetric extension of the Korteweg-de Vries equation, J. Math. Phys. 29 (1988), 24992506.

[14] Matveev V.B., Salle M.A., Darboux transformations and solitons, Springer Series in Nonlinear Dynamics, Springer-Verlag, Berlin, 1991.

[15] Nijhoff F.W., Discrete systems and integrability, Math3491/5491 Lecture Notes, University of Leeds, 2010.

[16] Nimmo J.J.C., Darboux transformations from reductions of the KP hierarchy, in Nonlinear Evolution Equations \& Dynamical Systems: NEEDS'94 (Los Alamos, NM), World Sci. Publ., River Edge, NJ, 1995, 168-177, solv-int/9410001.

[17] Roelofs G.H.M., Kersten P.H.M., Supersymmetric extensions of the nonlinear Schrödinger equation: symmetries and coverings, J. Math. Phys. 33 (1992), 2185-2206.

[18] Suris Yu.B., The problem of integrable discretization: Hamiltonian approach, Progress in Mathematics, Vol. 219, Birkhäuser Verlag, Basel, 2003.

[19] Xue L.L., Levi D., Liu Q.P., Supersymmetric KdV equation: Darboux transformation and discrete systems, J. Phys. A: Math. Theor. 46 (2013), 502001, 11 pages, arXiv:1311.0152.

[20] Zhou R.G., Private communication, 2013. 